\title{
DISFONIA E DISFUNÇÃO TEMPOROMANDIBULAR: HÁ RELAÇÃO?
}

\section{Dysphonic voice and temporomandibular dysfunction: is there any relation?}

\author{
Aline Tenório Lins Carnaúba ${ }^{(1)}$, Cristiane Cunha Soderini Ferracciu (2), \\ Érika Henriques de Araújo Alves da Silva ${ }^{(3)}$, Adriana Ricarte ${ }^{(4)}$, \\ Ana Carolina Rocha Gomes Ferreira ${ }^{(5)}$
}

\begin{abstract}
RESUMO
Objetivo: verificar se existe relação entre disfonia e disfunção temporomandibular (DTM). Métodos: este estudo foi realizado com 21 indivíduos, do gênero feminino, com faixa etária variando entre 18 - 27 anos, que não referiram sintomas de DTM, antes de serem apresentados ao questionário de triagem e, após o mesmo terem apresentado pelo menos um sintoma. Foram excluídos os indivíduos que não concordaram com o termo da pesquisa, não concluíram o protocolo de coleta de dados e os que apresentaram história de traumas e/ou cirurgia de face. A seleção dos sujeitos ocorreu de forma não probabilística por conveniência, utilizando-se pacientes que foram submetidos à triagem da Clínica Prof. Jurandir Bóia Rocha da Faculdade de Fonoaudiologia de Alagoas apresentando como queixa alterações vocais, levando em consideração os critérios de inclusão. Foram realizadas as seguintes avaliações: Fonoaudiológica (perceptivo-auditiva da Voz e específica para DTM) e Odontológica. Após os dados obtidos serem caracterizados com a utilização de técnicas de estatística descritiva, foi aplicado o Teste de correlação bivariada. Os cálculos foram obtidos através do software SPSS, na versão 16.0. Resultados: dos indivíduos com DTM: $46,15 \%(\mathrm{~N}=6)$ apresentaram qualidade de voz soprosa; 30,76\% ( $\mathrm{N}=4)$ articulação travada; $23,07 \%(\mathrm{~N}=3)$ loudness reduzida e $23,07 \%(\mathrm{~N}=3)$ com ressonância alterada. Conclusão: não houve correlação entre alterações vocais e disfunção temporomandibular, provavelmente devido ao número reduzido de sujeitos avaliados.
\end{abstract}

DESCRITORES: Distúrbios da Voz; Voz; Dor Facial; Transtornos da Articulação Temporomandibular

(1) Aluna de Graduação da Faculdade de Fonoaudiologia de Alagoas, UNCISAL, Maceió, AL, Brasil.

(2) Fonoaudióloga clínica; Professora Assistente da Faculdade de Fonoaudiologia de Alagoas, UNCISAL, Maceió, AL, Brasil; Mestre em Nutrição pela Universidade Federal de Pernambuco.

(3) Fonoaudióloga clínica; Professora Assistente da Faculdade de Fonoaudiologia de Alagoas, UNCISAL, Maceió, AL, Brasil; Especialista em Motricidade Oral pela Universidade Federal de Pernambuco.

(4) Fonoaudióloga clínica; Centro Avançado de Otorrinolaringologia e Cirurgia Estética facial - SINUS, Maceió, AL, Brasil; Especialista em Voz pelo Centro de Estudos da Voz.

(5) Fonoaudióloga; Professora Auxiliar da Faculdade de Fonoaudiologia de Alagoas, UNCISAL, Maceió, AL, Brasil; Especialista em Motricidade Oral pelo CEFAC - Pós-Graduação em Saúde e Educação.

Conflito de interesses: inexistente

\section{INTRODUÇÃO}

Disfunção temporomandibular (DTM) é um termo coletivo usado para descrever uma série de problemas clínicos que envolvem os músculos da mastigação, as articulações temporomandibulares (ATM) e estruturas associadas, ou ambos ${ }^{1-4}$.

Dentre os sinais e sintomas da DTM, encontram-se: dores nos músculos da mastigação ou na ATM, ruídos articulares, limitação da abertura oral, oclusão inadequada; sintomas otológicos como otalgia, plenitude auricular, zumbido e vertigem; cefaleias e sensibilidade em toda musculatura do sistema estomatognático e cervical ${ }^{5-11}$. Raramente estes ocorrem separadamente, mas sim como sintomas associados ${ }^{12}$. A dor e os ruídos articulares 
estão entre os sinais e sintomas mais complexos e frequentes ${ }^{13}$.

Estas disfunções são desencadeadas por uma combinação de fatores que incluem distúrbios da oclusão, distúrbios das bases ósseas (maxilar e mandibular); fatores traumáticos, problemas degenerativos, alterações musculares como hiperatividade ou hipoatividade, modificações funcionais e hábitos nocivos que levam a sobrecarga persistente na ATM ou na musculatura, estresse, e problemas emocionais ${ }^{14}$.

Por esta razão podemos observar que a DTM é uma das desordens mais complexas do organismo, capaz de desencadear alterações nos movimentos mandibulares, que provocam prejuízos tanto na articulação da fala como na qualidade da voz ${ }^{15,33}$. A voz, meio através do qual a fala é viabilizada, resulta da transformação do som gerado pelas pregas vocais ao longo do trato vocal e se modifica de acordo com a situação e o contexto da comunicação, através de ajustes motores precisos. Quando a harmonia muscular ao longo do trato vocal é mantida obtémse um som produzido sem desconforto pelo falante e de boa qualidade para o ouvinte. A ocorrência de desequilíbrios musculares durante a produção da voz gera o que é conhecido como disfonia, ou seja, a incapacidade de produzir a voz naturalmente e de transmitir a mensagem verbal de forma efetiva ${ }^{16}$.

Sendo assim, a associação dos problemas de voz e a "articulação travada" ou reduzida movimentação da mandíbula durante a fala, características da DTM, podem sobrecarregar a laringe, ocasionando a disfonia ${ }^{17,18}$. A redução nos movimentos mandibulares decorrentes desta alteração pode ser considerada como fator desencadeante das alterações vocais, principalmente quando há hipertonicidade contínua dos músculos supra-hióides. ${ }^{18-20}$.

Em uma pesquisa, realizada na Clínica de Fonoaudiologia da Universidade Estadual de Ciências da Saúde de Alagoas - UNCISAL, foi investigada a presença de sintomas de DTM em portadores de disfonia funcional e organofuncional, por meio de avaliações perceptivo-auditiva e específica para DTM, a fim de verificar se indivíduos disfônicos apresentavam sintomas de DTM. Os resultados revelaram que $9412 \%$ possuíam pelo menos um sintoma de DTM, com maior predominância de cefaleia e mordida desconfortável para alimentos duros e $88,4 \%$ apresentaram queixas audiológicas com prevalência de perda de equilíbrio seguido da presença de zumbido. As autoras concluíram que os indivíduos com disfonia apresentavam sintomas de DTM, devidos aos ajustes motores realizados por estes para manter o equilíbrio funcional, sendo assim predispostos a desenvolver tal disfunção ${ }^{19}$.
Foi observado, desta forma, um número expressivo da presença dos sintomas de DTM em indivíduos disfônicos o que despertou para a necessidade da realização de novas pesquisas, dando continuidade na investigação da correlação entre disfonia e DTM. É importante ressaltar que, na literatura em geral, poucos são os estudos que relacionam DTM e voz, e nem mesmo apontam a presença de sinais desta disfunção nas alterações vocais.

Sabendo-se da falta de literatura fonoaudiológica neste assunto, torna-se de interesse clínico e científico verificar se existe relação entre disfonia e DTM.

\section{MÉTODOS}

Este trabalho foi previamente aprovado pelo Comitê de Ética em Pesquisa da Universidade Estadual de Ciências da Saúde de Alagoas - UNCISAL, sob o protocolo de número 518.

Participaram deste estudo 21 sujeitos, do gênero feminino, com faixa etária variando entre $18-27$ anos, que não referiram sintomas de DTM, antes de serem apresentados ao questionário de triagem e, após o mesmo terem apresentado pelo menos um sintoma. Foram excluídos desta pesquisa indivíduos que não concordaram com o termo da pesquisa, não concluíram o protocolo de coleta de dados e os que apresentaram história de traumas e/ou cirurgia de face.

A seleção dos sujeitos ocorreu de forma não probabilística por conveniência, utilizando-se pacientes que foram submetidos à triagem da Clínica Prof. Jurandir Bóia Rocha da Faculdade de Fonoaudiologia de Alagoas da UNCISAL apresentando como queixas alterações vocais, levando em consideração os critérios de inclusão.

Para todo e qualquer procedimento realizado foi utilizado um termo de consentimento livre e esclarecido previamente aprovado pelo Comitê de Ética em Pesquisa da instituição de origem seguindo as preocupações éticas exigidas na realização de experimentos e avaliações em seres humanos, conforme a resolução 196/96 da Comissão Nacional de Ética em Pesquisa

Inicialmente foi realizada a aplicação de um questionário de triagem (Figura 1), contendo questões relativas às queixas de sinais e sintomas de DTM, considerando: presença de sintomas em repouso, abertura e fechamento oral, como dor, limitação dos movimentos mandibulares e desvio da mandíbula; queixas audiológicas (perda auditiva, zumbido, sensação de plenitude auricular e zumbido) e presença de hábitos orais deletérios sendo assim, possível selecionar a amostra. 


\section{Questionário de Identificação de Sinais e sintomas de DTM}

DADOS DE IDENTIFICAÇÃO:

№ do Protocolo:

Idade:

D.N.

Sexo: Profissão:

QUESTIONÁRIO:

1- Presença de sintomas em repouso, abertura e fechamento oral:

( ) Dor Região/ lado:

( ) Ruído: ( ) estalo ( ); crepitação ( )

( ) Saltos

( ) Cefaléia

( ) Dificuldades à mastigação, fala ou bocejo

( ) Limitação dos movimentos mandibulares (abertura de boca, lateralidade)

( ) Desvio da mandíbula

( ) "Travamento" da mandíbula

( ) Mordida desconfortável se restringindo a mastigar alimentos muito duros

( ) Sensibilidade à palpação

2- Queixas audiológicas:

( ) Zumbido ( ) OD ( ) OE

( ) Dor (otalgia) perto ou dentro dos ouvidos ( ) OD ( ) OE

( ) Sensação de plenitude auricular ( ) OD ( ) OE

( ) Perda auditiva ( ) OD ( ) OE

( ) Perda de equilíbrio

3- Possui hábitos deletérios?

( ) Bruxismo cêntrico noturno e/ ou diurno ( apertamento)

( ) Bruxismo excêntrico noturno e/ ou diurno ( rangimento)

( ) Onicofagia (roer unhas)

( ) Dorme com a mão apoiada no rosto

( ) Morde lábios, bochechas e/ ou objetos

( ) Masca chiclete

( ) Quebra gelo com os dentes

( ) Sucção digital

( ) sucção de objetos

( ) Outros:

4- Há quanto tempo sente essas dores?

( ) Menos de 1 ano

( ) 1 ano

( ) Mais de 1 ano

5- Já teve algum traumatismo na cabeça, pescoço ou mandíbula?
( ) Sim ( ) Não
( ) menos de 1 ano
( ) de 1 ano a 2 anos e 11 meses
( ) mais de 3 anos Em que região?

Figura 1 - Questionário de identificação de sinais e sintomas de DTM 
Em seguida, foram realizadas as seguintes avaliações:

1 - Avaliação específica para DTM baseada no protocolo de Bianchini (2004) ${ }^{14}$ (Figura 2), realizada por uma fonoaudióloga com especialização em motricidade orofacial, a fim de realizar um diagnóstico funcional para presença de DTM. Para a investigação e diagnóstico de DTM foi realizado exame clínico constituído por inspeção intra e extra-oral, palpação dos músculos mastigatórios(temporal anterior, médio e posterior, masseter superficial e profundo, pterigóideo medial) e sobre os que fornecem apoio secundário, como os do pescoço (esternocleidomastóideo, trapézio, cervicais posteriores) e das ATMs. Análise da movimentação mandibular, com utilização do paquímetro para medidas de máxima abertura oral e estetoscópio para a verificação de ruídos articulares. Para as avaliações funcionais (mastigação, deglutição e respiração), foi realizada filmagem, utilizando as consistências líquida (equivalente a água), pastosa (iogurte) e sólida (pão tipo francês de 25 gramas), assim como mensuração da aeração nasal através do uso do espelho milímetrado.

2 - Avaliação Odontológica para identificação de DTM, realizada por um cirurgião dentista, na qual constou de observação e palpação das estruturas e funções relacionadas com a ATM. Durante esta investigação, os sujeitos também eram submetidos à investigação da presença de má oclusão e desgaste dentário, para confirmação dos dados encontrados no questionário de índice anamnésico.

3 - Avaliação Vocal baseada no protocolo de Behlau $(2001)^{16}$ (Figura 3), realizada por uma fonoaudióloga especialista em voz, utilizando como instrumentais o programa grava som e filmagem. A mesma amostra vocal serviu simultaneamente para avaliação segundo a escala GRBASI e para análise perceptivo-auditiva. O grau global de disfonia foi avaliado a partir de cinco fatores: $\mathrm{R}$ (roughness) rugosidade, B (breathness) - soprosidade, A (asteny) - astenia, S (strain) - tensão e I - instabilidade. A amostra para a avaliação perceptivo-auditiva foi analisada seguindo os parâmetros: loudness (adequado, elevado e reduzido), tipo de articulação (precisa, imprecisa e travada), pitch (grave, agudo e adequado) e ressonância (laríngea, laringofaríngea, faríngea, hiponasal, hipernasal e equilibrada).

Após os dados obtidos serem caracterizados com a utilização de técnicas de estatística descritiva, foi aplicado o Teste de correlação bivariada, onde foi avaliado o grau de relacionamento linear através do coeficiente de Spearman para correlacionar a presença de DTM e as alterações vocais em indivíduos sem diagnóstico prévio de DTM. Os cálculos foram obtidos através do software SPSS, na versão 16.0 e os resultados serão considerados como significativos quando os valores de $p$ forem menores que 0,05 .

\section{RESULTADOS}

O levantamento do questionário de triagem revelou que a distribuição dos hábitos orais deletérios no estudo foi: $19,04 \%(\mathrm{~N}=4)$ bruxismo, $19,04 \%$ $(\mathrm{N}=4)$ onicofagia, $38,04 \%(\mathrm{~N}=8)$ dorme com a mão apoiada sobre o rosto, $38,04 \%(\mathrm{~N}=8)$ morde lábios, bochechas; $23,80 \%(\mathrm{~N}=5)$ quebra gelo com os dentes e $33,33 \%(\mathrm{~N}=7)$ têm o hábito de mascar chiclete.

Dos 21 voluntários que participaram da pesquisa, $61,90 \%(\mathrm{~N}=13)$ apresentavam disfunção temporomandibular, dos quais 15,38\% ( $\mathrm{N}=2)$ disfunção extra - articular bilateral, 30,76 \% (N=4) disfunção intra - articular unilateral e 53,84\% (N=7) disfunção intra - articular bilateral.

De acordo com a avaliação especifica para DTM, baseada no protocolo de Avaliação Miofuncional,

\section{Exame Fonoaudiológico da Articulação Temporomandibular}

\section{DADOS DE OBSERVAÇÃO}

(Bianchini, 2004)

1 - Características respiratórias

2 - Verificação das estruturas e musculatura

3 - Movimentos Mandibulares

4 - Dor a palpação extra-oral

Temporal/ Masseter/ ATM / Região orbitária/ Região zigomática/ Região submandibular/

Esternocleidomastoideo/ Região cervical/ Trapézio/ Região peitoral

5 - Funções estomatognáticas: Respiração/ deglutição/ mastigação / articulação

Figura 2 - Exame fonoaudiológico da articulação temporomandibular 


\section{Avaliação Perceptual - Auditiva}

(Behlau, 2001)

\section{ESCALA: GRBASI}

Emissão de sons:

- T.M.F: /a/

/e/

/i/

/u/

/s/

$|z|$

Relação s/z

- Pitch:

- Ressonância

- Loudness:

Qualidade Vocal:

- Tipo:

- Grau de Alteração:

Articulação:

- Tipo:

Velocidade da Fala:

Postura Coporal:

Figura 3 - Protocolo de avaliação perceptual-auditiva

46,15\% ( $\mathrm{N}=6)$ dos indivíduos portadores de DTM apresentaram padrão mastigatório bilateral simultâneo, $15,38 \%(\mathrm{~N}=2)$ bilateral alternado e $38,46 \%$ $(\mathrm{N}=5)$ unilateral. Conforme as características respiratórias, $53,84 \%(\mathrm{~N}=7)$ dos sujeitos apresentaram respiração nasal e 46,15\% (N=6) mista. Com relação à sensibilidade de dor a palpação extra-oral foi observada a prevalência de dor na região do masseter no feixe profundo (Tabela 1).

Os parâmetros da avaliação perceptivo-auditiva da voz (qualidade vocal, ressonância, loudness e articulação), nos indivíduos com DTM, apresentaram-se da seguinte forma: $46,15 \%(\mathrm{~N}=6)$ indivíduos com qualidade vocal soprosa, 30,76\% ( $\mathrm{N}=4)$ articulação travada, 23,07\% (N=3) loudness reduzida e $23,07 \%(\mathrm{~N}=3)$ com ressonância alterada. Para uma melhor visualização, os resultados serão apresentados na Tabela 2 e os achados posteriormente discutidos.

O teste de correlação bivariada utilizando o grau de coeficiente linear de Spearman revelou que não houve significância estatística para os dados correlacionados: tipos de alterações vocais e presença de DTM (Tabela 3).

\section{DISCUSSÃO}

Neste estudo optou-se por incluir apenas os indivíduos do gênero feminino, já que a disfunção temporomandibular acomete mais as mulheres que os homens, na faixa etária de 18 a 52 anos, indo ao encontro da literatura ${ }^{21-23}$.

Durante a análise dos protocolos de triagem, verificaram-se queixas diferentes entre pacientes, confirmando o que a literatura oferece sobre DTM e sua etiologia multifatorial ${ }^{24}$.

Os resultados do questionário sobre os hábitos orais deletérios corroboram com a literatura, afirmando que 1000 (mil) indivíduos entrevistados relataram possuir pelo menos um dos hábitos orais deletérios, sendo o hábito de maior incidência o apertamento dos dentes, apoiar o queixo nas mãos, dormir com a mão sob o rosto, pressionar a língua contra os dentes e morder os lábios ${ }^{25}$.

Com relação à palpação na musculatura extraoral verificou a presença de dor na musculatura mastigatória, concordando a literatura pesquisada que afirma que sujeitos com DTM apresentam movimentos mandibulares mais restritos e dor da musculatura mastigatória, devido ao acúmulo de carga proveniente de hábitos parafuncionais sobre as estruturas do sistema estomatognático 26-28.

Além das queixas de dor, os pacientes com DTM muitas vezes têm movimentos mandibulares limitados, ou assimétricos, e os sons da ATM descritos com mais frequência são cliques ou crepitações, o que também foi verificado neste estudo ${ }^{29}$. 
Tabela 1 - Distribuição dos portadores de DTM quanto à sensibilidade de dor à palpação extra-oral

\begin{tabular}{ccccccc}
\hline SUJEITOS & \multicolumn{5}{c}{ REGIÃO DE DOR À PALPAÇÃO } \\
\cline { 2 - 6 } & $\begin{array}{c}\text { Região de } \\
\text { Masseter - } \\
\text { feixe } \\
\text { profundo }\end{array}$ & $\begin{array}{c}\text { Região de } \\
\text { ATM }\end{array}$ & $\begin{array}{c}\text { Região de } \\
\text { Trapézio }\end{array}$ & $\begin{array}{c}\text { Região } \\
\text { Orbitária }\end{array}$ & $\begin{array}{c}\text { Região } \\
\text { Cervical }\end{array}$ & Sem dor \\
\hline 1 & $\mathrm{X}$ & $\mathrm{X}$ & $\mathrm{X}$ & & \\
2 & $\mathrm{X}$ & $\mathrm{X}$ & $\mathrm{X}$ & & \\
3 & $\mathrm{X}$ & $\mathrm{X}$ & $\mathrm{X}$ & & \\
4 & $\mathrm{X}$ & $\mathrm{X}$ & & \\
5 & $\mathrm{X}$ & $\mathrm{X}$ & & & \\
6 & $\mathrm{X}$ & $\mathrm{X}$ & & & \\
7 & $\mathrm{X}$ & $\mathrm{X}$ & & & \\
8 & $\mathrm{X}$ & & & & \\
9 & $\mathrm{X}$ & $\mathrm{X}$ & & \\
10 & & & & & \\
11 & & & & & \\
12 & & & & & \\
13 & & & & & \\
\hline
\end{tabular}

Tabela 2-Distribuição dos portadores de DTM quanto à escala GRBASle avaliação perceptivo-auditiva

\begin{tabular}{ccccccccccc}
\hline SUJEITOS & G & R & B & A & S & I & PITCH & RESSONÂNCIA & LOUDNESS & ARTICULAÇÃO \\
\hline $\mathbf{1}$ & 0 & 0 & 0 & 0 & 0 & 0 & Adequado & Equilibrada & Adequado & Precisa \\
$\mathbf{2}$ & 1 & 0 & 1 & 0 & 0 & 0 & Adequado & Equilibrada & Adequado & Precisa \\
$\mathbf{3}$ & 0 & 0 & 0 & 0 & 0 & 0 & Adequado & Faríngea & Adequado & Precisa \\
$\mathbf{4}$ & 0 & 0 & 0 & 0 & 0 & 0 & Adequado & Equilibrada & Adequado & Precisa \\
$\mathbf{5}$ & 1 & 0 & 1 & 0 & 0 & 0 & Adequado & Equilibrada & Reduzido & Travada \\
$\mathbf{6}$ & 1 & 0 & 1 & 0 & 0 & 0 & Adequado & Equilibrada & Adequado & Precisa \\
$\mathbf{7}$ & 0 & 0 & 0 & 0 & 0 & 0 & Adequado & Equilibrada & Reduzido & Precisa \\
$\mathbf{8}$ & 0 & 0 & 0 & 0 & 0 & 0 & Adequado & Laringofaríngea & Adequado & Precisa \\
$\mathbf{9}$ & 1 & 0 & 1 & 0 & 0 & 0 & Adequado & Equilibrada & Reduzido & Travada \\
$\mathbf{1 0}$ & 0 & 0 & 0 & 0 & 0 & 0 & Adequado & Equilibrada & Adequado & Travada \\
$\mathbf{1 1}$ & 1 & 0 & 1 & 0 & 0 & 0 & Adequado & Equilibrada & Adequado & Travada \\
$\mathbf{1 2}$ & 1 & 0 & 1 & 0 & 0 & 0 & Adequado & Laringofaríngea & Adequado & Precisa \\
$\mathbf{1 3}$ & 0 & 0 & 0 & 0 & 0 & 0 & Adequado & Equilibrada & Adequado & Precisa \\
\hline
\end{tabular}

Tabela 3 - Correlação entre alterações vocais e presença de DTM

\begin{tabular}{lcc}
\hline $\begin{array}{l}\text { Teste de Correlação } \\
\text { Bivariada }\end{array}$ & Sig (p) & R \\
\hline Qualidade Vocal & 0,387 & 0,199 \\
Articulação & 0,410 & $-0,190$ \\
Loudness & 0,086 & 0,384 \\
Ressonância & 0,404 & $-0,192$ \\
\hline
\end{tabular}

Sig. $(2$-tailed $)=$ valor de $\mathrm{p}$; Correlation Coefficient $=$ valor de $r$
De acordo com a avaliação miofuncional, $46,15 \%(\mathrm{~N}=6)$ dos indivíduos portadores de DTM apresentaram padrão mastigatório bilateral simultâneo, discordando dos achados da literatura que afirma ser mais comum realizarem mastigação unilateral ${ }^{30}$. Conforme as características respiratórias, $53,84 \%(\mathrm{~N}=7)$ dos sujeitos apresentaram respiração nasal, também não corroborando a literatura estudada que afirma prevalência do tipo respiratório oral. Esse valor de discordância pode 
existir em decorrência do grupo pesquisado ter sido de diferentes regiões climáticas ${ }^{31}$.

$\mathrm{Na}$ literatura pesquisada só foi encontrado um estudo que avaliasse a voz de indivíduos portadores de DTM segundo a escala GRBASI. Neste estudo observou-se um resultado semelhante aos dados da avaliação perceptivo-auditivo da presente pesquisa, apesar de seis indivíduos serem classificados com o tipo de voz alterada, a maioria deles apresentou alteração em pelo menos um dos parâmetros analisados (loudness, articulação e ressonância) ${ }^{32}$.

Dos parâmetros da avaliação perceptivo-auditiva da voz, nenhum apresentou relação estatisticamente significante com a DTM. Este achado contradiz os da literatura, que menciona a loudness como único parâmetro que apresentou relação estatisticamente significante ${ }^{33}$. Porém, outros afirmam que a loudness é um dos parâmetros que menos sofre alteração em portadores de DTM ${ }^{25,32}$.

A qualidade vocal mais encontrada no estudo foi do tipo soprosa, o que não corrobora os achados da literatura, que relatam a rouquidão como alteração vocal mais presente entre os indivíduos com DTM ${ }^{30-33}$. Porém, indo ao encontro do estudo de Pontes ${ }^{34}$.

Com relação à articulação, observou-se que três indivíduos com alteração vocal apresentavam articulação do tipo travada, evidenciando pesquisas realizadas que identificaram a articulação do tipo restrita e travada como as mais frequentes nessa população ${ }^{32,35}$.

Com relação à ressonância, os resultados obtidos nesse estudo podem ser justificados pelo fato de que nos indivíduos com DTM seja comum encontrar redução da atividade mandibular e isso pode levar a uma maior adução das pregas vocais. Com a adução inadequada das pregas vocais, o sistema ressonantal entra em desequilíbrio, deixando a ressonância com foco laringofaríngeo ${ }^{25,27}$.

\section{CONCLUSÃO}

Conclui-se com os resultados encontrados que:

Não houve correlação entre alterações vocais e disfunção temporomandibular, provavelmente devido ao número reduzido de sujeitos avaliados.

\section{AGRADECIMENTOS}

A Fundação de Amparo a Pesquisa de Alagoas - FAPEAL, pelo apoio financeiro.

\begin{abstract}
Purpose: to check whether there is a relationship between dysphonia and temporomandibular disorders (TDM). Methods: this study was conducted with 21 individuals, females, with ages ranging from $18-27$ years, who did not report TMD symptoms, before being submitted to the screening questionnaire and, after they had submitted at least one symptom. Subjects who did not complete the protocol for data collection and who had history of trauma and / or surgery of the face, were excluded from the study. The selection of the subjects occurred in a non-probabilistic form for convenience, using patients who underwent screening in the Clinic Prof. Jurandir Bóia Rocha of Faculdade de Fonoaudiologia of Alagoas, featuring vocal complaints, taking into account the inclusion criteria. The following assessments were carried out: Speech (perceptual Voice and specific TMD) and dental plan. After analyzing the data by using descriptive statistical techniques, we applied the bi-variation correlation test. The assessments were obtained using SPSS software, version 16.0. Results: within the subjects with TMD: $46.15 \%(\mathrm{~N}=6)$ had a breathy voice; $30.76 \%(\mathrm{~N}=4)$ joint locked; $23.07 \%$ $(\mathrm{N}=3)$ reduced loudness and $23.07 \%(\mathrm{~N}=3)$ with resonance changed. Conclusion: there was no correlation between vocal changes and temporomandibular dysfunction, probably due the number of evaluated subjects.
\end{abstract}

KEYWORDS: Voice Disorders; Voice; Facial Pain; Temporomandibular Joint Disorders 


\section{REFERÊNCIAS}

1. Okeson JP. Joint intracapsular disorders: diagnostic and nonsurgical management considerations. Dent Clin North Am. 2007; 51(1):85-103.

2. Biasotto-Gonzalez DA, Andrade DV de, Gonzalez TO, Martins MD, Fernandes KPS, Corrêa JCF, et al. Correlação entre disfunção temporomandibular, postura e qualidade de vida. Rev Bras Crescim Desenvolv Hum. 2008; 18(1):79-86.

3. Delgado VE, Schimist JE, Carlson CR, Leeuw RD, Okeson JP. Psychological and sleep quality differences between chronic daily headache and temporomandibular disorders patients. Cephalalgia. 2004; 24:446-54.

4. Bonjardim RL, Gavião MBD, Pereira LJ, Castelo PM. Mandibular moviments in childrem with or without signs and symptons of temporomandibular disorders. J Appl Oral Sci. 2004; 12(1):39-44.

5. Pereira KNF. Sinais e sintomas de pacientes com disfunção temporomandibular. Rev. CEFAC. 2005; 7(2):221-8.

6. Taucci RA. Verificação da interferência das disfunções temporomandibulares na articulação da fala: queixas e caracterização dos movimentos mandibulares [dissertação]. Rio de Janeiro (RJ): Universidade Veiga de Almeida; 2006.

7. Piozzi R, Lopes FC. Desordens temporomandibulares: aspectos clínicos e guia para a odontologia e fisioterapia. J. Bras. Oclusão, ATM Dor Orofacial. 2002; 2(5):43-7.

8. Melo GM. Disfunções temporomandibulares e dores orofaciais: uma visão interdisciplinar para o tratamento. Rev Dentística [periódico on line] 2008; 8(17). [acesso em 30 de julho de 2008] Disponível em: URL: http://www.ufsm.br/dentisticaonline

9. CostaLFM, GuimarãesJP, ChoabasA. Prevalência de distúrbios da articulação temporomandibular em crianças e adolescentes brasileiros e sua relação com má-oclusão e hábitos parafuncionais: um estudo epidemiológico transversal - Parte II: distúrbios articulares e hábitos parafuncionais. J Bras Ortodont Ortop Facial. 2004; 9(50):162-9.

10. Molina O, Mazzetto M, Stechaman J, Santos JR, Fernandes R, Pizzo R, et al. Distúrbios internos articulares. Retrodiscite em pacientes com Bruxismo e DCM-Características clínicas, diagnóstico e sugestões para tratamento. J Bras Oclusão, ATM e Dor Orofacial. 2001; 1(1):67-75.

11. Stegenga $B$, Schouten $H$. Mandibular pain and movement disorders, focusing on the temporomandibular joint. Ned Tijdschr Tandheelkd. 2007; 114(1):41-6.
12. Mcneill C. Temporomandibular disorders: diagnosis, management, education, and research. J Am Dental Assoc. 1990; 120:255-60.

13. Garcia AR, Souza V. Relação entre oclusão dentária e desordens temporomandibulares. Rev Fac Odontol Lins. 2001; 13(2):50-8.

14. Bianchini EMG. Articulação temporomandibular e fonoaudiologia. In: Piccolotto L, Befi-Lopes D, Limongi S. Tratado de fonoaudiologia. São Paulo: Rocca; 2004. p. 315-29.

15. Felício CM. Fonoaudiologia nas desordens temporomandibulares. Uma ação educativa. São Paulo: Pancast; 1994.

16. Behlau M. Conceito de voz normal e classificação das disfonias. In: Behlau M. Voz: o livro do especialista. Rio de Janeiro: Revinter; 2001. p.64-8.

17. Bianchini EMG. Relações das disfunções da articulação temporomandibular com a articulação da fala. Rev Dental Press Ortodont Ortop Facial. 2000; 5(1):51-9.

18. Blinchini EMG. Verificação da interferência das disfunções da ATM na amplitude e velocidade do movimento mandibular durante a fala por meio da eletrognatografia. Rev Dental Press Ortodont Ortop Facial. 2003; 8(3):109-15.

19. Silva EHAA, Ferracciu CCS, Rocha AC, Pedrosa FA. Sintomas de DTM em portadores de disfonia funcional e organofuncional. In: Anais do XIII Congresso Brasileiro de Fonoaudiologia; 2005; Santos: Revista da Sociedade Brasileira de Fonoaudiologia - Suplemento Especial; 2005.

20. Cookman S, Verdoline K. Interrelation of mandibular and laryngeal functions. J Voice. 1999; 13(1):11-24.

21. Cozzolino FA, Rapoport A, Franzi SA, Souza RP, Pereira CAB, Dedivitis RA. Correlação entre os achados clínicos e imaginológicos nas disfunções temporomandibulares. Radiol Bras. 2008; 41(1):13-7.

22. Nilsson IM, List $T$, Drangsholt M. Incidence and temporal patterns of temporomandibular disorder pain among Swedish adolescents. J Orofac Pain. 2007; 21(2):127-32.

23. Zhao YP, Ma XC. Temporomandibular disorders related pain interaction with age, sex and imaging changes of osteoarthrosis. Zhonghua Kou Qiang Yi Xue Za Zhi. 2006; 41(12):757-8.

24. Hernandes RC, Abalo RG, Mantín FC. Associação das variáveis oclusais e a ansiedade na disfunção temporomandibular. J Bras Oclusão, ATM e Dor Orofacial. 2001; 1(2):135.

25. Rodrigues L, Lemos J, Tokura M, Luz JGC. Freqüência de hábitos parafuncionais e suas manifestações clínicas em pacientes com 
disfunções da articulação temporomandibular. Rev. Odontol. UNICID. 2001; 13(2):113-23.

26. Bataglion C, Coronatto EASde, Bataglion A, Zuccolotto MCC, Chaguri NA, Menezes FB. Estudo da amplitude de movimentos mandibulares em pacientes com disfunção temporomandibular após a utilização de placa oclusal miorrelaxante. RPG - Revista de pós-graduação da Faculdade de Odontologia da Universidade de São Paulo. 2003; 10(1):19-24.

27. Celic R, Jerolimov V, Zlataric DK. Relationship of slightly limited mandibular movements to temporomandibular disorders, Ribeirão Preto. Brazilian dental journal. 2004; 15(2):151-4.

28. Branco RS, Branco CS, Tesc RS, Rapoport A. Frequência de relatos de parafunções nos subgrupos diagnósticos de DTM de acordo com os critérios diagnósticos para pesquisa em disfunções temporomandibulares (RDC/TMD). Rev. Dent. Press Ortodon. Ortop. Facial. 2008; 13(2):61-9.

29. Bove SRK, Guimarães AS, Smith RL. Caracterização dos pacientes de um ambulatório de disfunção temporomandibular e dor orofacial. Rev Latino-am Enferm. 2005; 13(5):686-91.
30. Parsinato F, Corrêa ERC, Peroni, ABF. Avaliação respiratória em indivíduos com DTM. Rev. Bras Fisioter. 2006; 10(3):285-9.

31. Felício CM, Melchior MO, Silva MAMR, Celeghini RMS. Desempenho mastigatório em adultos relacionado com a desordem temporomandibular e com a oclusão. Pró-Fono. 2007; 19(2):151-8.

32. Ferreira THP, Silva HJ, Balata PMM. Análise acústica e perceptivo-auditiva da voz na disfunção temporomandibular. Int J Dent. 2008; 7(4):212-8.

33. Silva ANT, Morisso MF, Cielo CA. Relação entre 0 grau de severidade de disfunção temporomandibular e a voz. Pró-Fono. 2007; 19(3):237-336.

34. Pontes PAI, Vieira VP, Gonçalves MJR, Pontes AAL. Características das vozes roucas, ásperas e normais: análise acústica espectográfica comparativa. Rev Bras Otorrinolaringol. 2002, 2:182-8.

35. Bianchini EMG. Avaliação fonoaudiológica da motricidade oral: articulação temporomandibular e fonoaudiologia. In Bianchini EMG, organizadora. Articulação temporomandibular: implicações, limitações e possibilidades fonoaudiológicas. Carapicuíba: Pró-Fono; 2000. p. 7-18.

DOI: 10.1590/S1516-18462010005000077

RECEBIDO EM: 29/11/2009

ACEITO EM: 12/04/2010

Endereço para correspondência:

Aline Tenório Lins Carnaúba

Rua Rubens Vilar de Carvalho, 47

Maceió - AL

CEP: 57052-606

E-mail: alinel_tenorio@ @otmail.com 